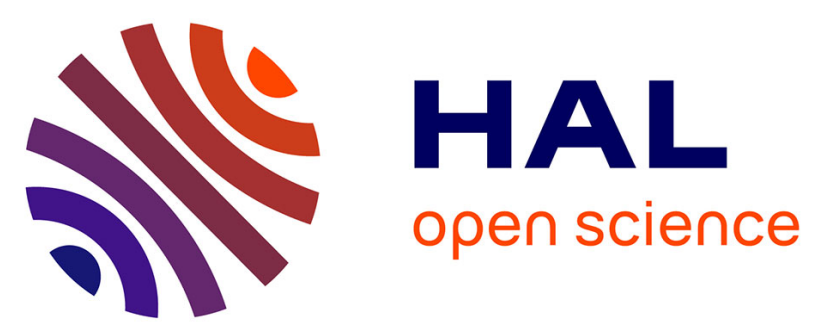

\title{
Les services publics de radio-télévision dans les DOM à l'ère du numérique: continuité des discours enchanteurs à propos de la "proximité", du "local" et de "l'ouverture sur le monde". Le cas de Réunion 1ère (France Télévisions)
}

Bernard Idelson

\section{To cite this version:}

Bernard Idelson. Les services publics de radio-télévision dans les DOM à l'ère du numérique: continuité des discours enchanteurs à propos de la "proximité", du "local" et de "l'ouverture sur le monde". Le cas de Réunion 1ère (France Télévisions). Les Enjeux de l'information et de la communication, 2013, 14/2, pp.135-148. hal-01501155

\section{HAL Id: hal-01501155 \\ https://hal.univ-reunion.fr/hal-01501155}

Submitted on 23 May 2017

HAL is a multi-disciplinary open access archive for the deposit and dissemination of scientific research documents, whether they are published or not. The documents may come from teaching and research institutions in France or abroad, or from public or private research centers.
L'archive ouverte pluridisciplinaire HAL, est destinée au dépôt et à la diffusion de documents scientifiques de niveau recherche, publiés ou non, émanant des établissements d'enseignement et de recherche français ou étrangers, des laboratoires publics ou privés. 


\title{
Les services publics de radio-télévision dans les DOM à I'ère du numérique : continuité des discours enchanteurs à propos de la "proximité", du "local" et de "l'ouverture sur le monde". Le cas de Réunion Ière (France Télévisions)
}

\author{
Bernard Idelson \\ Bernard Idelson est maître de conférences en sciences de l'information et de la communication à l'Université de \\ La Réunion. Au sein du Laboratoire de recherche sur les espaces Créoles et Francophones (LCF-EA 4549), il \\ s'intéresse à l'histoire et aux transformations des journalismes de l'océan Indien. \\ http://www.lcf-reunion.fr/equipe/idelson-bernard
}

\section{Résumé}

La télévision réunionnaise est inaugurée en 1965 par le ministre de l'Information, Alain Peyrefitte. Depuis, chaque nouveauté technique $\left(2^{\mathrm{e}}\right.$ chaîne, régie couleur, transmission des programmes par satellites, etc.) est l'occasion de discours enchanteurs sur le rôle que promet de jouer cette télévision à La Réunion. L’arrivée du numérique, avec notamment la Télévision numérique terrestre (TNT) en novembre 2011, s'inscrit dans cette représentation moderniste et techniciste. À travers l'analyse d'un corpus de discours officiels ou non - tenus par les décideurs de l'audiovisuel réunionnais durant sa courte histoire, il s'agit démontrer comment ces itérations discursives mettent à mal l'idée de " révolution numérique ». Car le processus de changement du Paysage audiovisuel réunionnais (PAR) est surtout lié aux mutations sociales, politiques et économiques à l'œuvre depuis quarante-cinq années.

Mots clés : Radio-télévision régionale - Médias numériques - Technicisme -La Réunion.

\section{Abstract}

The continuity of enchanting speeches about the "proximity", the «local " and « the outward looking attitude». The case of Réunion $1^{\text {ère }}$ (France Télévisions)

Reunionese television is launched in 1965 by the Minister of Information, Alain Peyrefitte. Since then, each technical innovation (2nd channel, control room, broadcast of programmes by satellites, etc.) is an opportunity to express « enchanting words » upon the role which this television promises to play in Reunion Island. The installation of digital technology, particularly the Digital Terrestrial TV in november 2011, comes within the scope of this modernist representation and this technical determinism. Through the analysis of a corpus composed of speeches - official or not - held by reunionese decision makers from the media during its short history, the purpose is to show how these discursive recurrences invalidate the idea of "digital revolution ". In fact, the process of change of the reunionese broadcasting scene is above all related to the social, political and economic transformation in progress since forty five years. 
Keywords: Regional radio-television - Digital media - Technical determinism - Reunion Island.

\section{Resumen}

Continuidad de los discursos espléndidos acerca de la «proximidad », de lo « local » y de la « apertura al mundo ». El caso de Réunion $1^{\text {ère }}$ (France Télévisions)

La televisión de la isla de Reunión fue inaugurada en 1965 por el ministro de Información, Alain Peyrefitte. Desde entonces, cada novedad técnica $\left(2^{\circ}\right.$ canal, televisión en color, transmisión de los programas por satélites, etc.) es motivo de discursos espléndidos sobre el papel que promete desempeñar la televisión en Reunión. La llegada de la era numérica, especialmente con la Televisión numérica terrestre (TNT) en noviembre de 2011, se inscribe en esta representación modernista y tecnicista. A través del análisis de un corpus de discursos - oficiales o no - asumidos por los decidores del sector audiovisual de Reunión durante su breve historia, se tratará de mostrar cómo esta repeticiones discursivas impactan en la idea de "revolución numérica ». Pues el proceso de cambio del Paisaje audiovisual reunionés (PAR) va vinculado, especialmente, con las mutaciones sociales, políticas y económicas que se verifican desde hace cuarenta y cinco años.

Palabras clave: Radio-televisión regional - Medias numéricos - Tecnicismo - Reunión

\section{INTRODUCTION}

Avec plusieurs années de décalage par rapport à la métropole, le lancement de la radio publique réunionnaise (RDF-Réunion) au lendemain de la Seconde Guerre mondiale, puis celui de la télévision (ORTF-Réunion), en 1965, ont été accompagnés de discours officiels et enchanteurs. Il était alors question de promettre à ces territoires un «avenir meilleur». Ainsi, lors des inaugurations des stations d'outre-mer, le ministre de l'Information de l'époque, Alain Peyrefitte, présenta la télévision d'outre-mer comme un objet de "progrès». Ces installations outre-mer s'inséraient dans un plan de réforme de l'audiovisuel, consacré notamment à la régionalisation, sur l'ensemble du territoire national. Le ministre entendait par là que ce nouveau média était destiné à apporter "culture, éducation et distraction », ou encore qu'il se présentait comme « une fenêtre sur le monde»(Idelson, 2012). Pourtant, dans les années qui suivirent, l'audiovisuel public domien apparut davantage comme une entreprise de contrôle politique, à visée assimilationniste, que comme un outil de renforcement du lien social ou du débat démocratique (idem, 2006). Il fallut attendre la libéralisation des ondes de la décennie 1980 pour observer l'émergence d'un espace public local médiatique (Watin, 2001). Nous proposons de montrer comment les discours actuels sur le numérique - légitimants et technicistes - s'inscrivent dans la continuité d'une représentation «moderniste », propre aux décideurs et aux programmeurs, du rôle de cet audiovisuel public réunionnais.

L'article s'articule en deux parties. La première soumet un cadrage théorique synthétique et contextualisé ; la télévision réunionnaise s'insère dans un espace public médiatique local en émergence. Quelques précisions méthodologiques sont également apportées concernant le sens donné dans ce travail au terme "discours»(1). La seconde présente l'analyse d'un corpus de discours de décideurs et de promoteurs et tente de les confronter avec l'environnement politico-médiatique dans lesquels ils sont énoncés. (2). La conclusion 
souhaite dégager quelques pistes de réflexion autour du thème de la continuité de ces discours, continuité qui met à mal l'idée de "révolution numérique » présentée, une nouvelle fois, comme salvatrice.

\section{UNE TELEVISION AU SEIN D'UN ESPACE PUBLIC MEDIATIQUE EN EMERGENCE}

Nous nous intéressons à une télévision insérée dans un contexte régional et aux discours de ses responsables sur son rôle. Pour cette raison, nous nous situons dans un cadre théorique à deux volets, l'espace public médiatique local (Watin 2001) et la formation discursive (Foucault, 1969) qui s'y rapportent. Cette approche conceptuelle permet ainsi d'analyser un média propre, mais en le considérant comme un élément à articuler dans une sociohistoire, non moins particulière, celle d'un Département d'outre-mer français (Dom), excentré d'un centre européen. Les configurations socio-politiques qui se jouent entre La Réunion et la métropole font partie intégrante de la compréhension de cette télévision réunionnaise qui appartient, malgré son évolution singulière, à l'ensemble de la télévision régionale française.

\section{Analyse de discours, analyse des discours}

Nous analysons un corpus composé de discours d'acteurs médiatiques ou de décideurs politiques qui donnent à voir leur représentation de la télévision. Ce n'est donc pas le discours produit par la «machine médiatique ", ni le «discours de l'information" (Charaudeau, 1997, p. 16 ; 2005, p. 28) qui est ici pris en compte, mais des paroles, des conversations, des déclarations qui circulent au sein ou en dehors de l'espace médiatique au sujet des médias. Ces discours mobilisés par des acteurs sont considérés comme des discours "en action ». C'est-à-dire qu'ils sont agrégés à des événements (ou du moins considérés comme tels par ces acteurs) médiatisés : inauguration des stations de télévision, innovations techniques, passage à la Télévision numérique terrestre (TNT) étayé par une campagne de communication. Ils peuvent avoir un caractère public ou émaner d'entretiens de recherche sur les lieux professionnels de production.

Les paroles réunies dans le corpus forment un ensemble discursif et sont recueillies dans des situations d'énonciations différentes : il peut s'agir d'entretiens ou d'allocutions télévisées. Certaines émanent d'acteurs politiques et constituent ainsi des discours politiques (au sens littéral du terme). Il convient donc de distinguer le terme générique de discours qui englobe l'ensemble des propos recueillis dans des situations diverses, de celui de discours officiels prononcés, par exemple à l'occasion d'une inauguration, ce dernier ne servant alors qu'à décrire le statut énonciatif des paroles recueillies. Il ne faut pas confondre non plus cette partie du corpus qui émane d'acteurs politiques (ministres inaugurant tel ou tel dispositif technique) du discours politique en général analysé sous un aspect macro-social (Le Bart, 1997). A contrario, notre analyse concerne des micro-occurrences observées dans l'espace social.

De même la notion de proximité repérée dans les discours des acteurs médiatiques n'est pas celle qu'utilisent les politistes (Le Bart, Lefebvre, 2005) : il ne s'agit pas de proximité politique, mais du réinvestissement discursif, par ces mêmes acteurs, d'un paradigme journalistique particulièrement présent à La Réunion depuis la libéralisation des ondes des années 1980 (Idelson, 2006 : 65). Les données présentées ont été sélectionnées en tant qu'isotopies discursives repérées au cours de la courte histoire de la radio et de la télévision réunionnaises. Leur analyse emprunte des procédés méthodologiques à des grilles d'analyse de discours (typologie argumentative, figures d'appui, etc.). issues de quelques- 
uns de nos travaux (référencés infra) et qui, compte tenu de la place impartie dans cet article, ne peuvent être présentés dans le détail.

In globo, les différences récurrences repérées sont considérées, au sens foucaldien, comme une formation discursive. Nous postulons que ces discours, même décelés aléatoirement, même "dispersés » au sein de l'espace social (dont celui des professionnels), révèlent une régularité de sens (Foucault, 1969, p. 53). Ils permettent en effet de dégager un ordre de valeur et de perception, partagé par les acteurs locaux ou nationaux, au sujet de la radio et de la télévision.

\section{Contextualiser des discours}

Ce faisant, il convient de contextualiser les périodes et les situations d'énonciation des discours produits à propos de la télévision.

La radio réunionnaise (station locale de la Radiodiffusion française - RDF) est lancée après la Seconde Guerre mondiale, mais se structure surtout en 1948, juste avant le changement institutionnel en Radiodiffusion-télévision française (RTF). Mais il faut attendre la transformation de la RTF en Office de radiodiffusion-télévision française (ORTF) pour qu'à la fin de l'année 1964, soit installée dans l'île une télévision, avec un Centre d'Actualités Télévisées (CAT). Cette implantation intervient au sein d'un contexte sociopolitique très tendu. En effet, au début des années 60, et malgré la départementalisation de1946, les infrastructures (sociales, sanitaires, routières, scolaires, etc.) sont encore nettement en deçà des besoins d'une population dont les représentants ont choisi une voie de décolonisation interne, au moment de la transformation du statut de l'île d'ancienne colonie en département. En pleine période de guerre froide, deux forces politiques s'opposent dans une bipolarisation reflétée dans la presse :les communistes locaux autour du Parti communiste réunionnais (PCR) de Paul Vergès, et les départementalistes autour de Michel Debré, élu député dans l'île en 1963. Ces derniers contrôlent la plupart des médias écrits et, bien sûr, l'unique radio d'État. Michel Debré présente le lancement de la télévision comme un «cadeau de Noël aux Réunionnais » destiné à «rompre l'isolement » (Debré, 1974, p. 49). Il s'agit pour lui d'étendre au DOM-TOM le plan de régionalisation de la télévision nationale débutée en France hexagonale, dans une logique générale du développement des informations infranationales. Depuis l'après Seconde Guerre mondiale, la Presse Quotidienne Régionale (PQR) poursuit par ailleurs son essor. Mais pour les opposants locaux (communistes et socialistes), l'audiovisuel réunionnais est considéré comme un instrument de propagande aux ordres de Paris, fonctionnant sur un mode hiératique. De fait, l'opposition locale autonomiste sera interdite d'antenne radio et télévision durant les périodes de l'ORTF et de FR3-Réunion. Il faudra attendre la création de RFO - la société de Radiodiffusion et de télévision Française pour l'Outre-mer est créée par décret le 17 septembre 1982 - pour que l'audiovisuel connaisse un certain pluralisme. Cette ouverture de l'espace public médiatique local se produit en fait par étapes et est liée à des mutations plus générales. La Réunion n'a pas connu de révolution industrielle, mais est passée rapidement (en une trentaine d'années) du mode de fonctionnement d'une société de plantation à celui d'une société du tertiaire, dans laquelle émerge une classe moyenne (petits fonctionnaires, entrepreneurs, commerçants). Durant la décennie 1970, les sphères urbaines, scolaires et médiatiques se transforment. Pour analyser la société réunionnaise, le modèle canonique habermassien de l'espace public bourgeois ne peut être convoqué qu'avec prudence et doit être revisité (Simonin, Watin, 1992). Ici, l'émergence de l'espace public est lié à cette classe sociale moyenne, demandeuse d'ouverture, et se manifeste dans plusieurs sphères lors de divers événements. Dans la 
sphère médiatique, ces étapes successives sont constituées par l'apparition d'une presse lycéenne au début des années 1970, la création du journal Le Quotidien de La Réunion et de l'océan Indien en 1976, et par l'éclosion des radios libres au début de la mandature présidentielle de François Mitterrand. Ce dernier point d'ouverture mérite une attention particulière, dès lors qu'on souhaite comprendre les logiques et les représentations actuelles des promoteurs des services publics de la radio-télévision réunionnaise.

En métropole comme à La Réunion, la fin du monopole radio a pour origine la $94^{\mathrm{e}}$ des 110 propositions énoncées en 1981 par le candidat Mitterrand: "La télévision et la radio seront décentralisées et pluralistes. Les radios locales pourront librement s'implanter dans le cadre du service public. Leur cahier des charges sera établi par les collectivités locales. Sera créé un Conseil national de l'audiovisuel où les représentants de l'État seront minoritaires" (D’Almeida, Delporte, 2003, p. 238-239).À La Réunion, porté par cette ouverture, le Docteur Camille Sudre, membre de l'Association pour la Libération des Ondes (ALO), lance Radio FreeDom, le 14 juillet 1981. Se démarquant fortement de la programmation de FR3-Radio Réunion, Radio FreeDom propose des émissions interactives, de proximité, intitulées «Les radios doléances ». Les auditeurs peuvent s'y exprimer sur les sujets de leur choix, en créole, parfois avec véhémence. Dans le domaine de l'information, l'ouverture est également effective grâce à un partenariat entre FreeDom et Europe $\mathrm{N}^{\circ} 1$ pour la diffusion de journaux en direct. Une tentative de collaboration avec les journalistes du Quotidien a lieu également pour proposer aux auditeurs un journal radiophonique local. D'autres stations locales diffusent des journaux nationaux : RFM passe des accords avec RTL (RMC sera relayée en 1991 par Radio Est).La Haute Autorité de la Communication Audiovisuelle (HACA) accordera ensuite une quarantaine de fréquences à des radios privées ou associatives. Cette succession de brèches médiatiques dans le paysage audiovisuel réunionnais ne sera pas sans effet sur la station locale de FR3 (radio et télévision) qui devient RFO-Réunion le $1^{\text {er }}$ janvier 1983. Ses responsables tentent alors de modifier l'image qu'ils pensent véhiculer, celle de relais d'un pouvoir centralisateur parisien (jusqu'aux années 1980, la station publique de radio et télévision clôt encore ses émissions, vers $23 \mathrm{~h}$, au son de la Marseillaise). Jusqu'à aujourd'hui, cette volonté affichée d'être un média de «proximité » au «service des Réunionnais » apparaît comme la matrice principale des discours et des programmations, y compris lorsqu'ils accompagnent la présentation de nouveautés techniques numériques.

Cette volonté trouve son origine dans ce que nous avons appelé le traumatisme des événements de 1991. Fort du succès d'audience de Radio FreeDom et des recettes publicitaires qu'elle commence à engranger, Camille Sudre lance Télé FreeDom, sans autorisation légale, le 13 mars 1986, entre les deux tours de l'élection législative. Le fondateur de FreeDom légitimise son action par la nécessité de "combattre le monopole de l'audiovisuel d'État» (Ponthus 1995, p. 91). Ce credo sera brandi à chaque manifestation de rue, qui mobilise plusieurs milliers de personnes, organisée par les partisans de FreeDom pour soutenir «leur» télévision. Mais plusieurs plaintes en justice sont déposées contre FreeDom. Et c'est finalement le projet Antenne-Réunion/Canal+ qui emporte l'un des appels d'offres lancés par le Conseil supérieur de l'audiovisuel, lequel organisme émettant de plus en plus de réserve sur FreeDom. Cette situation tendue aboutit, malgré les soutiens des élus locaux, à la saisie des émetteurs, ordonnée par le procureur de la République, le 24 février 1991. Un mouvement social, d'une rare intensité à La Réunion, s'ensuit, baptisé par les médias "Les événements du Chaudron ", (du nom du quartier de Saint-Denis dans lequel se produisent une partie des émeutes). Huit personnes sont tuées dans l'incendie d'un magasin. Ce mouvement social est également lié 
à la situation socio-économique dans laquelle se trouve alors La Réunion, département aux taux de chômage et d'allocataires du RMI (Revenu minimum d'insertion) les plus élevés de France. Isolé un moment de la classe politique, Camille Sudre reviendra néanmoins au devant de la scène politico-médiatique en se faisant élire président du conseil régional en 1992. Son élection sera invalidée l'année suivante, au motif que l'utilisation de Radio FreeDom à des fins électorales aurait empiétée sur les comptes de campagne. Mais Margie Sudre succédera à son mari à la présidence de la Région. Camille Sudre assumera les fonctions de vice-président de cette collectivité jusqu'en 2010.

Au cours de la décennie 1990-2000, l'offre de programmation s'intensifie et les canaux de diffusion se diversifient. Suite au lancement du satellite Eutelsat W2 (le 5 octobre 1998 par le vol 11 d'Ariane), deux nouveaux opérateurs de télévision satellitaire thématique (Canal Satellite et Parabole Réunion) élargissent considérablement le paysage audiovisuel réunionnais. RFO- Réunion doit faire face à une concurrence accrue au sein d'un marché publicitaire insulaire limité, et se positionner en termes d'image et de contenu. Il lui faut, par la diversification de sa programmation et le pluralisme de son information, convaincre ses auditeurs et ses téléspectateurs, qu'elle s'insère dorénavant dans ce nouvel espace public médiatique local. Face à l'interactivité et la représentativité des nouveaux médias locaux, des initiatives sont prises. L'Association des radios et des télévisions de l'océan Indien (Artoi) tente de mettre en ouvre une coopération régionale. L'arrivée du numérique (les premières connexions à l'internet dans l'île sont effectives en 1997) rend possible cette ouverture indo-océanique, qui reste néanmoins limitée, pour des raisons structurelles et institutionnelles : par exemple, les liaisons aériennes entre les différentes îles sont coûteuses et les différences de statuts politiques des États insulaires ne facilitent guère les accords de coopération. Des fonds sont débloqués pour moderniser les infrastructures techniques (studio d'enregistrement vidéo mobile, location d'un second canal satellite permanent, Telecom 2), tandis que des essais (pas toujours bien accueillis) de bulletins d'information en créole sont entrepris (Idelson, 2004).

Après la période de régulation des années 1990, RFO, dotée d'un budget propre, doit assumer une logique de concurrence d'audience. En juillet 2004, l'entreprise, toujours soumise à un cahier des charges de service public, est intégrée au groupe France Télévisions. Le paradigme de la proximité et du local est encore mis en exergue : RFO gère les programmes des radios et télévisions "pays » des neuf stations d'outre-mer. La société change à nouveau d'appellation et devient Réseau Outre-Mer $1^{\text {re }}$, le 16 mars 2010. Ce changement intervient à la suite d'une énième réforme de l'audiovisuel, la loi du 9 juillet 2004, relative aux communications électroniques et aux services de communication audiovisuelle. Les neuf stations sont désormais : Guadeloupe $1^{\text {ère }}$, Guyane $1^{\text {ère }}$, Martinique $1^{\text {ère }}$, Mayotte $1^{\text {ère }}$, Nouvelle Calédonie $1^{\text {ère }}$, Polynésie $1^{\text {ère}}$, Réunion $1^{\text {ère }}$, Saint-Pierre et Miquelon $1^{\text {ère }}$. La même année, deux initiatives reflètent cette volonté emblématique d'être «à la pointe » du numérique : la mise en place des matinales d'information diffusées en cross media (radio, télévision et internet) et l'arrivée de la TNT.

Cette mise en perspective généalogique et socio-historique s'avère ainsi nécessaire à la compréhension et à l'analyse du corpus de discours que nous présentons à présent. Depuis l'inauguration de la télévision réunionnaise jusqu'à celle de la TNT, les thèmes de "rattrapage »avec la métropole, de développement des infrastructures techniques et de proximité avec les usagers y sont récursifs et séquentiels. 


\section{L'ANALYSE DES DISCOURS DES DECIDEURS ET DES PROMOTEURS DE LA RADIO-TELEVISION PUBLIQUE}

Notre corpus analyse en diachronie des propos d'acteurs liés à la radio et à la télévision réunionnaises. Les statuts d'énonciation et de recueil de ces discours sont variés. Le terme discours peut être pris dans son sens littéral, on l'a évoqué, lorsqu'il s'agit de discours officiels tenus par un représentant du gouvernement. Les discours du corpus peuvent également émaner d'entretiens journalistiques (en plateau) ou de recherche. Cette étude s'inscrit dans des programmes menés en sciences de l'information et de la communication autour de l'espace public médiatique local. Nous avons sélectionné six discours d'acteurs correspondant à trois catégories (discours pionniers, discours inauguraux et officiels, discours managériaux). La période couverte est celle comprise entre l'installation de la télévision à La Réunion et l'arrivée récente de la TNT (qui permet de recevoir dix chaînes : Réunion $1^{\text {ère }}$, Antenne Réunion, le bouquet France Télévisions, Arte, France 24 et Télé Kréol) ${ }^{1}$.

\section{Le discours d'un acteur pionnier, Jean Vincent-Dolor ${ }^{2}$}

Premier speaker-rédacteur de la radio publique réunionnaise, premier rédacteur en chef du Journal de l'Ile de la Réunion, puis du bureau d'information la radio et de la télévision (RTF-ORTF, FR3-Réunion), correspondant de l'AFP, durant quarante ans, J.V.-D. aura une action déterminante sur l'audiovisuel réunionnais. Dans le cadre d'études sur le journalisme réunionnais, une biographie sociale (sociobiographie) de cet acteur clef, mémoire du paysage médiatique réunionnais, a été retracée (Idelson, 2012). Ancien résistant, gaulliste convaincu, J.V.-D. est très proche du pouvoir debréiste. Il présente le premier bulletin d'information de la télévision réunionnaise en janvier 1965, au moment de son inauguration officielle par le ministre Peyrefitte. Quelques clefs significatives du discours de J.V.-D., à propos de l'audiovisuel réunionnais ainsi que de la société réunionnaise de l'après-départementalisation, permettent d'appréhender une certaine vision des médias d'information et de distraction propre aux décideurs de cette période.

\section{Le discours inauguraux : Alain Peyrefitte, Rémy Pflimlin, Marie-Luce Penchard}

Deux moments d'inauguration, considérés comme emblématiques, ont été retenus en raison de la mise en perspective comparative qu'ils permettaient. Quarante-cinq années les séparent en effet. Le premier discours est celui prononcé par Alain Peyrefitte, au moment du bulletin d'information diffusé lors de l'inauguration de la télévision réunionnaise le 31 janvier $1965^{3}$. Avec l'emphase et le lyrisme caractéristiques des discours officiels de la période, le ministre présente les atouts pour l'île qu'il voit dans l'arrivée de ce nouveau medium.

Le 29 novembre 2010, la station de Réunion $1^{\text {ère }}$ inaugure l'arrivée de la TNT, dispositif qui propose de recevoir gratuitement dix chaînes de télévision (contre vingt en métropole). Une intervention de la ministre chargée de l'outre-mer, Marie-Luce Penchard, est diffusée ${ }^{4}$.

\footnotetext{
${ }^{1}$ Dans la place impartie, nous nous limitons seulement ici à synthétiser quelques résultats de notre recherche.

${ }^{2}$ Désormais J. V.-D.

${ }^{3}$ Discours transcrit dans l'édition du 2 février 1965 du journal La Liberté, et dans celle du 4 février 1965 de la Démocratie.

${ }^{4}$ La ministre reprend également les principaux thèmes de son intervention dans une interview accordée au Quotidien de la Réunion (édition du 29/11/2010).
} 
La ministre s'adresse aux téléspectateurs face à la caméra, dans la tradition d'intervention officielle. Il ne s'agit cependant que d'une séquence de l'opération d'autopromotion cérémonielle organisée par la station de La Réunion. En effet, au cours de cette soirée télévisée, Réunion $1^{\text {ère }}$ fait également intervenir plusieurs acteurs médiatiques ou politiques, sous forme d'interviews réalisées par ses propres journalistes. Le dispositif choisi est alors celui de l'entretien (faussement?) décontracté, presque intimiste. Plusieurs responsables de la société Outre-Mer $1^{\text {ère}}$, dont son président Rémy Pflimlin, se succèdent ainsi dans les studios, en tant qu'acteurs d'autorité de cette célébration.

\section{Les discours managériaux : Benoit Saudeau et Robert Moy}

$\mathrm{Au}$ moment de l'inauguration de la TNT, le directeur régional de Réunion $1^{\text {ère, }}$ Benoit Saudeau, est sur le départ. Il sera remplacé par Robert Moy. Leur propos, tenus lors d'entretiens individuels, sur la TNT et sur la situation de la station qui subit une forte concurrence, révèlent également leurs représentation du rôle du média qu'ils dirigent.

Trois items principaux - isotopiques - ressortent alors de l'analyse de l'ensemble de ces discours. Ils sont marqués par des itérations de valeurs et de conception de la télévision, qui, somme toute, ne varient pas beaucoup au cours de la période concernée. Il s'agit de l'isotopie de la modernité, de celle du progrès, et de celle de la proximité avec l'usager, qui célèbre le fait local sans exclure, non plus, l'ouverture sur le monde.

\section{L'appel à la " modernité »}

La notion de modernité apparaît récurrente dans les discours tenus à propos de La Réunion, qui a longtemps été considérée comme un territoire dans lequel devait se réaliser un "rattrapage » structurel - en termes d'équipements - avec la métropole. Historiquement et politiquement, la revendication originelle de la départementalisation va dans ce sens : elle est issue du Comité Républicain Action Sociale (CRADS) qui réclame, à la Libération, un changement de statut pour La Réunion qui optera ainsi pour une "décolonisation intra-française " (Combeau, 2009, p. 145). Depuis, la référence à la modernité (incarnée par le modèle de la France métropolitaine) est partagée par tous les acteurs politiques. Elle est déjà présente au moment où ces protagonistes sont le plus divisés dans leur engagement à propos du statut de l'île, lors de l'inauguration de la télévision. Deux camps s'opposent alors localement : les départementalistes, partisans du député Michel Debré, qui contrôlent une grande partie des journaux ainsi que l'audiovisuel d'État, et les opposants proches du Parti communiste réunionnais, partisans d'une évolution de statut allant dans le sens d'une plus grande autonomie. Durant la période d'exercice de J. V.-D. à la tête du bureau d'information, le secrétaire général du PCR, Paul Vergès, à qui il est reproché de vouloir l'indépendance de l'île, n'apparaîtra jamais à la télévision. Il faudra attendre la transformation de la station de FR3-Réunion en RFORéunion, en 1983, pour que cette forme de censure politique cesse. Mais, tous les protagonistes de cette bipolarisation (nationalistes-départementalistes opposés aux autonomistes) prônent l'entrée de l'île dans une plus grande modernité.

${ }^{5}$ Les propos de Rémy Pflimlin et Marie-Luce Penchard proviennent d'interviews réalisées par des journalistes de Réunion $1^{\text {ère }}$ lors de l'inauguration de la TNT ; leur transcription a été effectuée par Manuela Gigant, dans le cadre de la préparation d'un Master Recherche en Sciences de l'information et de la communication, en cours, à l'Université de La Réunion. Les entretiens des directeurs régionaux Benoit Saudeau et Robert Moy ont également été réalisés et transcrits par ses soins. 
Cette récurrence injonctive de modernité ressemble fort à ce que M. Foucault appelle une "régularité discursive » qu'il décrit comme "déjà à l'œuvre dans chaque commencement, [et qui permet] de découvrir un principe de cohérence et l'esquisse d'une unité future, de maîtriser le temps par un rapport perpétuellement réversible entre une origine et un terme jamais donnés, toujours à l'œuvre» (Foucault, 1969, p. 32).Ainsi, les différentes transformations institutionnelles de l'île (départementalisation, accès au statut de région européenne, etc.) apparaissent souvent comme des mesures permettant de passer d'une tradition obsolète à une modernité prometteuse. Mais, à partir des années 1980, la notion de « respect de la culture locale » est également assumée. On peut repérer ce rapport au cours de l'histoire dans chaque discours d'acteur de la télévision, y compris dans les plus récents - par exemple à propos du numérique - avec, en filigrane, l'idée que l'État français, à travers le service public de la télévision, participe à cette avancée ${ }^{6}$. À quarantecinq années d'intervalle, les passages infra tirés du corpus, illustrent ces sèmes communs.

Alain Peyrefitte (31/01/1965) :

«[...] Cela, c'est la vie traditionnelle de votre île, comme c'était déjà la vie de l'île Bourbon. La télévision évoquera ce passé, vous fera mieux connaître la nature et les beautés de l'île, ranimera votre fierté. Mais depuis quelques années, votre département connaît également un prodigieux essor. La télévision doit suivre le rythme de ce département. Elle vous rendra compte de l'extraordinaire transformation que vont vous apporter à tous, année après année, de meilleurs salaires et un meilleur niveau de vie. [...]

La télévision vous fera suivre cette poussée de sève qui s'appelle la montée des jeunes. Elle vous fera participer à la vie nouvelle qui remplace progressivement la quiétude d'autrefois, grâce à une jeunesse active et ambitieuse, prête au travail, ici ou en métropole. »

Marie-Luce Penchard (21/11/2011) :

«[...] La TNT, c'est la télé de l'avenir [...]. L'État viendra aussi aider les ménages à s'équiper, en particulier ceux qui ont des revenus plus faibles, et nous avons mis en place un dispositif de communication et d'accompagnement qui s'appelle "Tous au numérique "[...]. La TNT, c'est un beau projet, à la fois à la pointe de la modernité, et qui permet une plus grande ouverture culturelle et sociale au ceur du pacte républicain. »

\section{Le progrès technique}

Si les promoteurs et acteurs de la télévision locale évoquent le progrès social, ils ne le dissocient guère du progrès technique. Cette surdétermination techniciste est également présente à toutes les époques de l'histoire de la télévision. Elle se retrouve dans le discours de J. V.-D. lorsqu'il se déplace en personne à Paris, au début des années 60, pour réclamer l'installation de la télévision à La Réunion. Il se heurte alors à une fin de non recevoir et manifeste une forte déception et rancœur à l'égard des «décideurs parisiens ». Mais, plus tard, il attribue l'installation de la télévision à La Réunion à Michel Debré, qui est élu député dans la $1^{\text {ère }}$ circonscription de l'île en 1963. Dans les discours de lancement de la télévision réunionnaise, les valeurs exprimées peuvent renvoyer au triptyque bien connu «distraire, informer, instruire»des fondateurs tels que Jean d'Arcy (Pierre, 2003, p. 58). Mais, à La Réunion, jusqu'aux années d'ouverture (comme en métropole durant la période

\footnotetext{
......

${ }^{6}$ Réunion 1 ère dispose en 2013 d'un budget de 18,5 millions d'euros, provenant à plus de $90 \%$ des ressources de la redevance et des contributions de l'État allouées à France Télévisions. Le reste est constitué des recettes publicitaires. (Source : http://reunion.lalere.fr).
} 
d'exercice d'Alain Peyrefitte), la télévision est surtout un instrument de contrôle (Idelson, 2012). Le discours techniciste occulte cette réalité politique. La nature de la programmation et les contenus ne correspondent pas toujours aux promesses inauguratoires.

Alain Peyrefitte (31/01/1965) :

«A $11000 \mathrm{~km}$ de Paris, isolée dans l'océan Indien, votre île, depuis Noël, dispose de la télévision. À l'heure où certaines régions montagneuses de la métropole ne reçoivent pas encore la télévision, Saint-Denis possède sa propre station.

En moins d'un an, sous l'impulsion de vos responsables et notamment de votre député, Michel Debré et, grâce aux prouesses réalisées par les techniciens de l'ORTF, toutes les installations ont été construites et mises au point. »

Rémy Pflimlin (21/11/2011) :

«(...) les enjeux (du numérique), c'est d'abord la capacité de développer une offre de proximité plus claire, plus autonome. C'est ensuite la possibilité pour le service public, de présenter l'ensemble de ses chaînes, à l'ensemble de nos concitoyens. ”

Marie-Luce Penchard (21/11/2011) :

"Aujourd'hui la télévision numérique terrestre arrive chez vous. La TNT, c'est la télé de l'avenir, celle qui permet de recevoir gratuitement un plus grand nombre de chaînes. C'est une véritable révolution du paysage audiovisuel ultramarin. "

Au cours de l'histoire de la radio et de la télévision réunionnaise, l'idée de performance technique accompagne chaque installation de nouvel équipement (régie couleur, télécinéma, satellite, etc.). Elle est reliée à un imaginaire de la technique (Flichy, 2001) qui occulte les autres déterminants socio-politiques évoqués en première partie. Au traumatisme des événements de 1991, s'ajoute également le fait que les médias concurrents de Réunion $1^{\text {ère, }}$ qui la devancent largement en termes d'audience, ne bénéficient pas, en raison de leurs budgets moindres, des mêmes infrastructures techniques.

\section{La célébration du fait local, de la " proximité ", mais aussi de "l'ouverture sur le monde »}

L'émergence de l'espace public médiatique réunionnais date des premières brèches médiatiques constituées par l'éclosion d'une presse de lycéens qui souhaitent débattre, dans leurs journaux, de la société locale (Wolff, 1998). Le succès d'audience de radio et de Télé FreeDom puis, à partir de 1991, d'Antenne Réunion (la télévision privée concurrente de Réunion $1^{\text {ère}}$ ) repose au départ sur des contenus de programmation qui laissent une large place au fait local et à la langue créole, relativement occultés jusqu'aux années 80 dans l'audiovisuel public. Depuis, les termes de "proximité » et de «local» («Radio et Télé pays») constituent des itérations enchanteresses, brandies par les responsables de la télévision à chaque opération de promotion de leurs actions. Désormais, la télévision publique doit participer au processus de patrimonialisation de la culture créole réunionnaise, même si cette vision se heurte à la programmation de contenus formatés, issus de l'extérieur, et aidant à se positionner en termes d'audience.

La métaphore de la télévision constituant une "fenêtre sur le monde » date du lancement de la télévision et est toujours utilisée pour présenter les transformations liées au numérique. 
Benoit Saudeau, directeur de Réunion $1^{\text {ère }}(9 / 03 / 2011)$ :

"[...] sur le créole, j'ai envie de dire qu'on n'est pas dans un combat, on est dans une évolution harmonieuse d'une situation qui effectivement évolue. Je crois que ces combats culturels sont assagis, moi je suis hanté par une crainte c'est que notre télé notre radio ne soit plus synchrone avec notre pays. Et à partir du moment où effectivement on perd la synchronisation, à partir du moment où on se désincarne, à partir du moment où on se sanctuarise en cessant d'exploiter les richesses, et le créole en est une, mais ça peut aussi constituer un obstacle, si on veut par exemple exporter nos produits, il faut qu'ils soient accessibles au plus grand nombre. À partir du moment où effectivement on fait attention à ne pas perdre la synchro, je crois qu'on a résolu une grande partie de nos problèmes. Vous n'avez pas connu le montage film, et la bande son qui était dé-synchro avec l'image du film, c'était ma hantise quand j'étais journaliste[...], culturellement c'est resté dans le reste de ma vie professionnelle, c'est resté vraiment un point de repère important. Si la radio et la télé que l'on fabrique ne sont plus les reflets maîtrisés de notre réalité culturelle historique économique sociale... C'est que nous sommes sur un faux rythme nous ne sommes pas dans le bon tempo.

[...]Voilà. Donc la TNT... C'est dix et puis bientôt peut-être encore sept ou huit chaînes qui seront lancées sur des bouquets, puis c'est une façon de rester au tempo du reste de la planète, voilà. »

Robert Moy, directeur de Réunion $1^{\text {ère }}$, succédant à B. Saudeau(12/03/2012) :

"La TNT ne va pas régler le problème de la taille du marché. Ici à La Réunion, c'est 850000 habitants, et la télévision reste également toujours, en tout cas la production locale, une industrie de main-d'œuvre qui coûte cher. [...]. Donc mettre en place des programmes locaux, il faut avoir la capacité de pouvoir le faire [...]. La seule chaîne qui a renforcé la production locale dans le bouquet TNT, depuis que la TNT a été lancée, c'est nous! ».

Rémy Pflimlin (21/11/2011) :

«Nous avons une carte très particulièrement à jouer, nous, le service public, qui est la carte d'une télévision qui est inscrite dans son territoire proche des gens avec de l'information, des magazines, avec aussi tout ce qui est en terme d'animation des partenariats, et puis l'ouverture sur le monde, l'Inde toute proche et l'océan Indien tout proche, bref faire en sorte que cette télévision corresponde à la vie des gens qui habitent La Réunion. »

\section{CONCLUSION}

Nous avons tenté de montrer comment ces discours légitimants, sélectionnés en diachronie, apparaissaient empreints d'un imaginaire techniciste et déterministe (Breton, Proulx, 1989 ; Flichy 1995, 2001). Nous postulons que cette représentation des principaux décideurs et acteurs de la radio et de la télévision des Dom perdure, voire se renforce, au fur et à mesure des changements d'appellation des organismes (ORTF, FR3, RFO, Réunion $\left.1^{\text {ère }}\right)$. Les mutations techniques du numérique - même si elles sont bien effectives en termes de facilité de diffusion- ne modifient guère plus les conceptions des programmeurs et leur vision des utilisateurs. Pour autant, l'arrivée de concurrents, depuis la privatisation de l'audiovisuel, contraint les radios et télévisions publiques à faire face à des enjeux économiques particulièrement âpres. 
Cette tension est la résultante de deux principaux facteurs : l'étroitesse du "marché » de cet audiovisuel insulaire ; la contradiction entre l'injonction qui lui est faite de s'insérer dans une logique de concurrence économique déréglementée et son cahier des charges fixant des missions de service public ${ }^{7}$.

Face à cette situation complexe, le discours techniciste est considéré une nouvelle fois comme salvateur. Pourtant l'installation de dispositifs techniques présentés comme « révolutionnaires » semble occulter les véritables changements sociétaux (Rebillard, 2007). Car, de facto, les transformations sont plutôt liées aux contextes de réception, aux pratiques et aux attentes des usagers (téléspectateurs, auditeurs, internautes) de ce service encore public, mais qui doit s'insérer dans une logique privée d'audiences.

Cette approche empirique concerne une situation «périphérique», par rapport à un centre européen. Elle permet de questionner des modèles canoniques (comme celui de l'espace public habermassien) issus de l'Europe du Nord, tout en incitant à la prudence concernant une éventuelle montée en généralités à propos de l'audiovisuel public national et de sa tradition (Pierre, 2012), ou de l'histoire générale de la télévision régionale (Lafon, 2012).

Enfin, faute de place, ces discours n'ont pas pu être confrontés aux contenus de la télévision; il aurait été par exemple intéressant de détailler l'évolution des programmations, notamment d'information. Mais leur analyse aura peut être révélé leur fonction d'accompagnement. Car, comme le souligne Armand Mattelart (2001, p. 109), " aucune pédagogie d'appropriation citoyenne du milieu technique ne peut s'abstraire de la critique des mots qui, prétendument apatrides, n'ont de cesse de faire intrusion dans le langage commun et d'encadrer les représentations collectives ».

Ainsi, l'analyse critique des discours des promoteurs de la télévision réunionnaise constitue un atout heuristique pour l'analyse des représentations qui circulent dans cet espace social local et par conséquent pour sa connaissance.

\footnotetext{
${ }^{7}$ Les directeurs régionaux successifs scrutent régulièrement les parts d'audience (PDA). Gilles Vaubourg a remplacé Robert Moy en octobre 2012, date depuis laquelle la station enregistre une légère hausse d'audience, bien qu'elle soit largement devancée par ses concurrents. Le Sondage Médiamétrie-Métridom (Vague avril-juin 2013 pour la télévision et Vague janvier-juin 2013 pour la radio) indiquait les résultats suivants :

- Télévision : Antenne Réunion : 28,0\%, Réunion $1^{\text {ère }}: 13,7 \%$,

- Radio : FreeDom : 33,6\%, Réunion1 ${ }^{\text {ère }}: 9 \%$ (Source : www.mediametrie.fr).

${ }^{8}$ Dans l'ouvrage qu'il consacre à l'histoire de la télévision régionale, Benoit Lafon prend soin de présenter distinctement « la régionalisation outre-mer» (Lafon, 2012, p. 81).
} 


\section{REFERENCES BIBLIOGRAPHIQUES}

Almeida (d'), Fabrice, Delporte, Christian, 2003, Histoire des médias en France, de la Grande Guerre à nos jours, Paris : Flammarion, coll. Champs Université.

Breton, Patrick, Proulx, Serge, 1989, L'explosion de la communication, Paris : La Découverte, coll. Manuel Repères, [4 éd. 2012].

Charaudeau, Patrick, 1997, Le discours d'information médiatique. La construction du miroir social, Paris : Nathan/Institut national de l'audiovisuel (INA), coll. Médias-Recherches.

Charaudeau, Patrick, 2005, Les médias et l'information. L'impossible transparence du discours, Bruxelles : De Boeck, coll. Médias recherches.

Combeau, Yvan, (s/d.), Lîlle de La Réunion dans le XXe siècle. Un itinéraire français dans l'océan Indien, Colonie, Département, Région, Saint-Denis de La Réunion :Éd. du Centre de recherches sur les sociétés de l'océan Indien (CRESOI).

Debré, Michel, 1974, Une politique pour la Réunion, Paris : Plon.

Flichy, Patrick, 1995, L'innovation technique. Récents développements en sciences sociales, vers une nouvelle théorie de l'innovation, Paris :La Découverte.

Flichy, Patrick, 2001, L'imaginaire d'internet, Paris : La Découverte.

Foucault Michel, 1969, L'archéologie du savoir, Paris : Gallimard, coll. nrf, Bibliothèque des sciences humaines.

Idelson, Bernard, 2004, "Le créole dans les médias réunionnais », Hermès, n 40, p. 128134.

Idelson, Bernard, 2006, L'histoire des médias à La Réunion, de 1946 à nos jours, Paris : Le Publieur.

Idelson, Bernard, 2012, "L'installation de la télévision dans les DOM : ouverture ou fermeture d'un espace public médiatique ? Le contre-exemple de La Réunion », in Pierre, Sylvie, (s/d.), La télévision, le temps des constructeurs,Pensée et stratégies d'un père fondateur,Paris : Ina/l'Harmattan, coll. Les médiasen actes, p. 93-108.

Lafon, Benoit, 2012, Histoire de la télévision régionale, de la RTF à la 3, 1950-2012, Bry-surMarne : Ina Éditions, coll. médias histoire.

Le Bart, Christian, Le discours politique, 1998, Paris : Presses Universitaires de France (PUF), coll. Que sais-je?

Le Bart, Christian, Lefebvre, Rémi, 2005, La proximité en politique. Usages, rhétoriques, pratiques, Rennes : Presses Universitaires de Rennes (PUR), coll. Res Publica.

Mattelart, Armand, 2001, Histoire de la société de l'information, Paris : La Découverte, coll. Repères.

Pierre, Sylvie, 2012, (s/d.), La télévision, le temps des constructeurs. Jean d'Arcy, pensée et stratégies d'un père fondateur, Paris : Ina/l'Harmattan,coll. Les médiasen actes.

Ponthus, Arnaud, 1995, Le phénomène FreeDom à l’Ile de La Réunion. Médias et politique. Rochemaure : Éd. Simone Sudre.

Rebillard, Franck, 2007, Le web 2.0 en perspective. Une analyse socio-économique de l'internet, Paris : L'Harmattan, coll. Questions contemporaines. 
Simonin Jacky, Watin, Michel, 1992, «L'espace public réunionnais : une opportunité pour problématiser certaines problématiques établies ", Les nouveaux espaces de l'information et de la communication,Actes $d u$ gème Congrès National des Sciences de l'Information et de la Communication, Lille :CRDO/SFIC, p. 407- 413.

Watin, Michel, 2001,(s/d.),Communication et espace public - Univers Créoles, $\mathrm{n}^{\circ} 1$, Paris :Anthropos-Économica.

Wolff, Éliane, 1998, Lycéens à la Une, Saint-André de La Réunion : Océan Éd. 